\title{
Aromatic Profile of Hydroponically and Conventionally Grown Tomatoes
}

\author{
Melina Korčok $^{1} \mathbb{D}$, Nikola Vietorisová ${ }^{1}$, Patrícia Martišová ${ }^{2} \mathbb{D}$, Jana Štefániková ${ }^{2} \mathbb{D}$, Anna Mravcová ${ }^{3}$ and \\ Vladimír Vietoris ${ }^{1, *}$
}

1 Department of Technology and Quality of Plant Products, Faculty of Biotechnology and Food Sciences, Slovak University of Agriculture in Nitra, Trieda A. Hlinku 2, 94976 Nitra, Slovakia; xkorcok@uniag.sk (M.K.); vietoris@uniag.sk (N.V.)

2 AgroBioTech Research Centre, Slovak University of Agriculture in Nitra, Trieda A. Hlinku 2, 94976 Nitra, Slovakia; patricia.martisova@uniag.sk (P.M.); jana.stefanikova@uniag.sk (J.Š.)

3 Department of Social Sciences, Faculty of Economics and Management, Slovak University of Agriculture in Nitra, Trieda A. Hlinku 2, 94976 Nitra, Slovakia; anna.mravcova@uniag.sk

* Correspondence: vladimir.vietoris@uniag.sk

Citation: Korčok, M.; Vietorisová, N.; Martišová, P.; Štefániková, J.; Mravcová, A.; Vietoris, V. Aromatic Profile of Hydroponically and Conventionally Grown Tomatoes. Appl. Sci. 2021, 11, 8012. https:// doi.org/10.3390/app11178012

Academic Editors: Davide Giacalone and Marek Dolezal

Received: 16 June 2021

Accepted: 25 August 2021

Published: 30 August 2021

Publisher's Note: MDPI stays neutral with regard to jurisdictional claims in published maps and institutional affiliations.

Copyright: (c) 2021 by the authors. Licensee MDPI, Basel, Switzerland. This article is an open access article distributed under the terms and conditions of the Creative Commons Attribution (CC BY) license (https:// creativecommons.org/licenses/by/ $4.0 /)$.

\begin{abstract}
Hydroponics is a more environmentally friendly and economical way of growing crops that allows crops to be grown all year round, regardless of soil and climate conditions. Hydroponic cultivation of various fruits, vegetables, flowers, etc., is well known and used today. Tomatoes also play an important role in hydroponic cultivation. Tomatoes grown in this way should have a more pronounced aroma and flavor, and the fact that they are harvested at the ripe stage should also be reflected in the fresh red color and appropriate organoleptic characteristics of the fruit. This study was concerned with the sensory analysis of hydroponically grown tomatoes (Solanum lycopersicum) compared with conventionally grown tomatoes of the same species. Samples were evaluated by instrumental sensory analysis using an electronic nose as well as a sensory panel. In this study, the difference between hydroponically and conventionally grown tomatoes was demonstrated. These differences were also captured by the consumers (sensory panel). When analyzing the odor profile of the samples, we found that hydroponic tomatoes are characterized by the presence of chemical organic compounds, namely: 2-methylpropanol, 2,3-pentanedione, and (Z)-3-hexen-1-ol or 1-hexanol, which cause the fruity aroma of the fruit. These substances are very likely to characterize the differences between the tested samples. The electronic nose has shown to be a potentially suitable tool for detecting differences and identifying typical product markers, which may suggest its further use in food authentication detection. It is also interesting to find almost no correlation between the two methods studied.
\end{abstract}

Keywords: aromachology; scents; behavior; aroma; hydroponics; conventional cultivation; tomato; sensory analysis; sensory panel; electronic nose

\section{Introduction}

Today's market offers us a wide choice of fruit and vegetables throughout the whole year. Consumers are increasingly paying attention to the quality of their food and sensory quality is becoming more and more important for consumer decision making when choosing a product. It is certainly an important factor when purchasing the same product again, where consumers mainly consider whether the product was of the required aroma and taste characteristics for them [1].

The flavor is a combined sensation of taste and aroma; sugars, acids, and volatile compounds are their main determinants [1]. A large number of volatile compounds have been identified and the volatile profile of tomato has been investigated closely in many studies [2-5]. The resulting flavor characteristic of tomato is the result of complex interactions between organic acids, sugars, and more than 400 volatile compounds. Of 
the volatile compounds identified, less than $10 \%$ are present at significant concentrations and at thresholds that are likely to affect the odor and taste of tomatoes. Several studies have shown that only 16 of the total aromatic volatiles are present in sufficient quantities to be detected by the olfactory system. Therefore, the following selected descriptors are recognized as carriers of tomato taste and are present in significant quantities in the fruit. However, minor volatiles with negative odor units should not be neglected as they may still contribute to the overall aroma as adjuvants [6,7]. The individual interactions between volatiles, also those involving the gustatory and olfactory systems are significant. These complicate the aroma because specific volatile aromas perceived by the retronasal olfactory system can influence the perception of sweetness or sourness and vice versa [8,9]. Tomato fruit aroma is important in determining consumer perception and acceptability of fruit products [1]. The aroma of fresh ripe tomato is mainly attributable to cis-3-hexenal, cis3-hexenol, hexanal, 1-penten-3-one, methyl salicylate, 2-isobutylthiazole and ß-ionone in appropriate concentrations. These compounds are biosynthetically derived from the degradation of compounds such as fatty acids, amino acids, carotenoids, and are also formed by other biosynthetic pathways [10].

In recent decades, the selection of tomato cultivars has emphasized parameters such as yield, fruit size, firmness, product flawlessness, disease resistance, ease of handling and longer shelf life, rather than sensory aspects of fruit quality. As a result, consumers started to complain about the poor tomato flavor [11-13]. Tomato flavor is now commonly referred to as "classic tomato flavor" or "old-fashioned tomato flavor", which indicate the deterioration of the sensory quality of commercial tomato fruits. However, it is unclear how, why and whether the quality of the fruit has actually changed, but consumers associate recent varieties with a lack of aroma, although such an association has not been scientifically proven. The fact is that there is now an increasing number of consumers who demand freshly grown products with a higher quality of aroma, flavor, and taste [1]. It is this recent problem, but also the demand for higher quality products, and the need of the market for year-round availability of tomatoes that stimulates the search for new ways of their growing.

Hydroponic growing systems offer an opportunity to at least augment the traditional in global food production. The advantages of hydroponic growing systems include the reduction of water wastage (recirculation), the ability to manipulate conditions to maximize production (according to [14], $\mathrm{NaCl}$ treatment which increases sweetness, sourness, umami (i.e., flavor deliciousness) and overall preference) in a limited space (vertical gardens), and the fact that crops are grown in a controlled environment (control of pests, nutrients, and attributes necessary for optimal plant growth). Hydroponics is becoming an increasingly popular way of growing crops, as it has several advantages compared to the traditional way of growing tomatoes [15].

In agriculture, hydroponic cultivation is a common cultivation method in greenhouses, or more precisely in hydroponic environments, where plants are grown in an inert substrate (such as perlite or rockwool) using crop fertilization (irrigation with a nutrient solution) [16] In a hydroponic system and in soil cultivation, before the elements are bioavailable to the plant, the inorganic and organic parts must be dissolved and decomposed in water. In soil-grown plants, the elements stick to the soil particles and pass into the soil solution where they can be absorbed by the roots of the plant. In hydroponically grown plants, the nutrient solution containing the elements comes into contact with the plant roots, where the roots can then take up the minerals and water [15].

According to some data, tomatoes represent the largest share of hydroponically grown crops, as this method of cultivation can produce red and juicy tomato fruits with a variety of flavors, colors and can contain health-promoting ingredients such as lycopene, which helps maintain a healthy cardiovascular system and provides a wide range of other health benefits. Verdoliva et al. [17] stated that hydroponically grown tomatoes had higher lycopene and $\beta$-carotene content compared to conventionally grown tomatoes. It is from these substances that the volatile compounds that contribute to the typical tomato aroma are produced metabolically. These authors report that they have been able to produce crops 
of similar or better quality in the hydroponic way compared to food grown in soil, using significantly less water. It is clear that hydroponic tomatoes can taste as good as tomatoes grown in rich soil outdoors, or even better $[18,19]$. Tomatoes grown in this way should have a more pronounced aroma and flavor, and the fact that they are harvested at the ripe stage should be reflected in the fresh red color and adequate sensory characteristics of the fruit. Although the hydroponic system has many advantages, it does not automatically guarantee a high-quality product. As the technology advances, it is important to consider the sensory characteristics of the hydroponic product [20].

The aroma of tomato has been the subject of research, and the two main techniques have been the most commonly used for aroma assessment-sensory analysis and conventional gas chromatography-mass spectrometry (GC-MS). These methods are generally time consuming and difficult. A good application of these methods is important to obtain adequate results. In recent years, electronic nose systems (e-nose) have been designed to address the need for routine quality testing in the food industry. Electronic nose devices are designed to simulate the human olfactory system and are devices that can detect and recognize odors and flavors using an array of sensors. The use of the e-nose consists in the analysis of volatile organic compounds and single emerging odors that are released as gases or vapors from solids or liquids. Since the day the first prototype was developed, the e-nose has become a useful device in several applications in solving urgent problem situations, finding relevance in various industrial fields such as healthcare, automotive, food industry, environmental monitoring, food storage, and even military industry. Especially in the food industry and medicine, traditional methods of object recognition are too slow, expensive, and mostly subjective, which can result in fatal mistakes. Instead, e-nose technology offers a fast, sensitive, low-cost, and objective alternative. Moreover, the potential of this new sensing method continues to grow rapidly with new developments in sensor and computer learning technologies [21-23].

There are many different types of tomatoes to choose from on the shelves of supermarket chains. There are different varieties, sizes, shapes, colors, different ways of growing tomatoes, whether conventional, organic, hydroponic or many others. Can consumers identify the difference between the offered tomatoes? Could hydroponically grown tomatoes really taste and smell differently from conventional ones? If so, what accounts for these differences? From these questions, the main objectives of the present study emerge, in which we focused on the characterization of the aromatic profile of hydroponically grown tomatoes. For this purpose, a sensory analysis of hydroponically grown tomatoes compared to conventionally grown fruits of the same species was designed and carried out. The evaluated hydroponically grown samples came from Slovakia and Czechia, while the conventionally grown samples came from abroad. Structurally, this study was divided into two parts. The first part dealt with the instrumental sensory analysis of tomato odor profiles using e-nose and the second part dealt with the determination of differences with a more detailed focus on flavor profiles between hydroponic and conventionally grown tomatoes through the sensory panel using profile methods. The study also focused on the determination of odorants typical for hydroponically grown tomato species.

\section{Materials and Methods}

\subsection{Sampling}

Ten types of cherry tomatoes grown either hydroponically or conventionally were sampled on the basis of a food market survey targeting wholesale chains. All samples were purchased at the same time and used Slovak and Czech hydroponically grown tomatoes compared to foreign conventional tomatoes. These samples came from the Netherlands, Belgium and Italy. The samples were labeled with a three-digit numerical code, and only code marking was used at all times. All the tomato samples evaluated were of first-class quality. In this case, we experimented on the basis of the products available on the market, so the samples differed in variety or were not described on the packaging.

A summary of the samples evaluated was given in the form of Table 1. 
Table 1. Overview of sensory evaluated samples.

\begin{tabular}{lll}
\hline \multicolumn{2}{l}{ Hydroponically Grown Tomatoes } & \\
\hline Sample Number & Country of Origin & Variety \\
\hline V269 & Slovak Republic & Unreported \\
V801 & Slovak Republic & Unreported \\
V153 & Czech Republic & Unreported \\
V512 & Czech Republic & Unreported \\
V931 & Slovak Republic & Tramezzino \\
V740 & Slovak Republic & Mc Dreamy \\
\hline Conventionally Grown Tomatoes & \\
\hline Sample Number & Country of Origin & Variety \\
\hline V297 & Belgium & Axiana \\
V102 & Netherlands & Sweetele \\
V188 & Netherlands & Dulcita \\
V826 & Italy & Dulcita \\
\hline
\end{tabular}

\subsection{Sample Preparation}

The study consisted of two parts. The first part used an electronic nose, which is a tool for analyzing organoleptic features using artificial perception. The second part focused on the evaluation of sensory properties using a sensory panel. After purchase, the samples to be evaluated using the electronic nose were allowed to rest and cure overnight at constant room temperature. These samples were scrape-free, washed and cut into small pieces, and $5 \mathrm{~g}$ of each sample was transferred into the appropriate vials according to the three-digit code. The filled vials were allowed to stand sealed for $2 \mathrm{~h}$ at room temperature in the laboratory and after this time, the samples were subjected to e-nose analysis. The samples for evaluation by the sensory panel were given in quantities of approximately $15 \mathrm{~g}$ and corresponded to the characteristics evaluated and the requirements for assessing the taste of the fruit, and therefore, each sample consisted of approximately one whole fruit and one half tomato fruit. During preparation, emphasis was placed on ensuring the homogeneity of the samples and compliance with the hygiene and sampling principles of ISO 8589 . Similar to the preparation of samples for e-nose evaluation, samples were labeled with the same three-digit code.

\subsection{Methods}

\subsubsection{Electronic Nose}

Ten tomato samples were analyzed using a Heracles II electronic nose (Alpha MOS, Toulouse, France), with three constant replicates taken from each species. The sample preparation procedure was described above and represented an average sample according to standard sampling procedures. Samples were mixed in a balanced random order in the tray and analysis was performed automatically using an autosampler. Data were processed with the native AlphaSoft 14.2 program.

\subsubsection{Sensory Panel Assessment}

In the second part of the study, sensory evaluation of tomato samples was carried out by a sensory panel in the sensory laboratory of the Slovak University of Agriculture in Nitra, Department of Hygiene and Food Safety. The sensory panel consisted of 12 evaluators in two sessions, which, in our case, represented a group of selected persons (according to ISO 8586:2007 standard). Suitable environmental conditions were ensured (average ambient temperature of $20^{\circ} \mathrm{C}$ at a relative humidity of approximately $65 \%$ ), as well as all appropriate environmental conditions relating to the room, lighting and avoidance of other objective factors that could influence the evaluation process. The samples were distributed to the evaluator in a random, balanced order. The evaluators were provided with a taste neutralizer and a five-minute rest interval. 
In the first part, the evaluators assessed the tomatoes from a more comprehensive point of view using a scoring test (including visual characteristics and texture).

The second part of the questionnaire consisted of evaluating the sensory profiles by observing what values the raters assigned to the samples on a $15 \mathrm{~cm}$ scale in the attributes of aroma and flavor and their subcategories. The basic flavor attributes evaluated were sour taste, sweet taste, and variations of ripe-overripe taste, metallic taste, spicy/sharp taste, and off-taste. Specific values were obtained by measuring the position of each sample on a given axis.

\subsection{Statistical Analysis}

Principal component analysis (PCA) was used to process the electronic nose results, where features with the greatest discriminatory power between samples were selected. For visualization, the native electronic nose program AlphaSOFT version 14.1 was used. For the purpose of predicting the growing patterns, we used the machine learning (ML) technique of the Caret of statistical software package R 4.0.2. Several algorithms (KNN, SVM, RF and LDA) were used; the results are available below. We used canonical correlation analysis (CCA) of the statistical software CCA package $\mathrm{R}$ version 4.0.2 to find the relationship between the sensory data and the electronic nose data.

The results obtained through the sensory panel were evaluated in RStudio version 3.6.3 using the PCA method and the SensoMiner package. The results are represented by standard descriptive indices (shown by boxplots). Pairwise comparison tables indicate the $p$-value after calculation by the non-parametric Friedman test.

\section{Results and Discussion}

\subsection{Electronic Nose}

The distribution of the samples based on the total odor profile is shown in Figure 1. This distribution was based on the volatile organic compounds released during the analysis process. We selected 12 major odorants based on discriminant strength relative to all observed samples. From this figure, we can see that samples V740 (SR), V102 (NL) and V269 (SR) have a volatile profile as they form a large triangle during the measurement time. We consider samples V297 (BE) and V512 (CZ) to be stable and least variable in the change of the odor profile. The other samples have an approximately constant odor spectrum, which means that they are almost equally extractive. The position of samples V102 (NL) and V269 (SR) suggests to us that these samples are similar. These two samples are also characterized by high odor content. Interestingly, these samples represent hydroponically (V269) and conventionally (V102) grown samples. The Slovak sample of hydroponically grown tomatoes (V801) also shows similarities with these two samples.

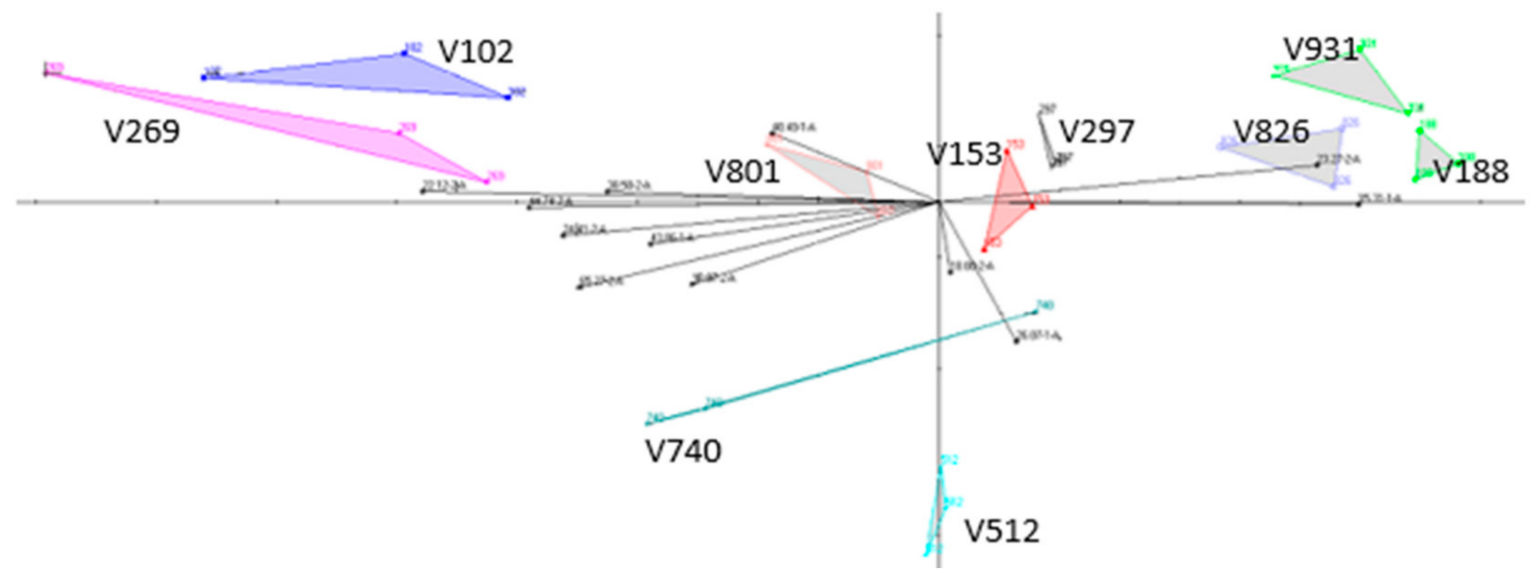

Figure 1. Total odor profile of samples with corresponding odors. 
Samples (V826, V931, V188) that are in the same quadrant are similar. These samples represent tomatoes that have been stripped of their top leaves in the processing and packaging process.

This figure also shows the odors captured by the e-nose during the analysis of the samples. Specific odorous substances produced by volatile organic compounds are represented by lines in the figure and their direction indicates the products that contain the most of them. At the ends of the lines are numbers used to identify the compounds that were present in the samples. We can confirm that samples V102 (NL), V269 (SR) and V740 (SR) are more extractive even though they contain fewer types of odorous compounds compared to the others. Odor compounds 26.07-1-A and 15.31.1-A (listed in Table 1) are typical of most hydroponically grown samples with strong discrimination power.

In the following procedure, we discussed the most basic identifiers that occurred in significant amounts in tomato samples grown hydroponically. From the total odor profile that was detected and generated from the electronic nose database, we selected the first five basic odor markers that occurred in the greatest amount in the samples through the program. Based on this, the localization and odor spectrum of the samples were changed. This is illustrated in Figure 2. Since $98 \%$ of the $100 \%$ results obtained were evaluated, we assess that the results obtained are reliable as well as quantitatively and qualitatively adequate.

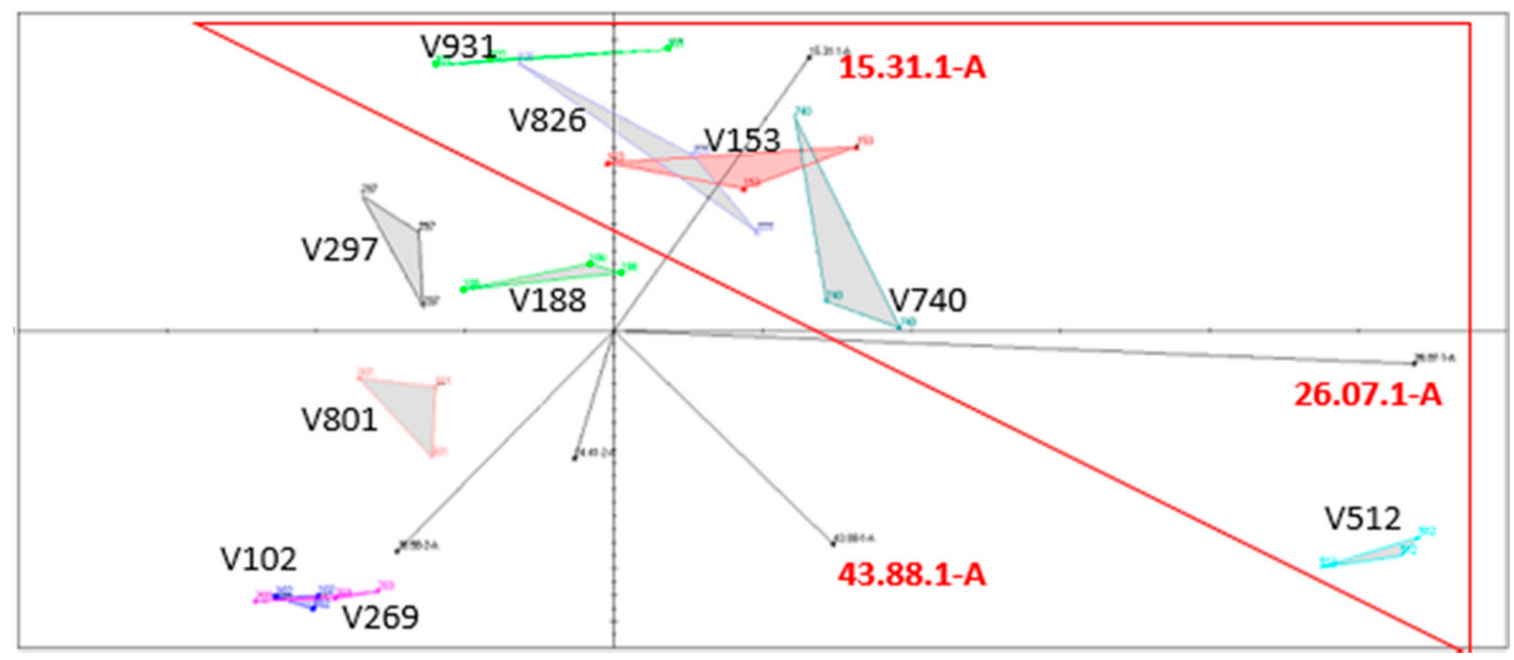

Figure 2. Categorization of samples with selection of main markers.

E-nose was able to identify differences between the evaluated samples and classify them into two groups: hydroponically and conventionally grown tomatoes. The device assessed the hydroponic tomatoes as demonstrably similar and placed them side by side on the graph. For better clarity, we marked all these samples in the form of a triangle. Bottom left quadrant represents the samples that the device assessed as conventionally grown. This includes sample V102 (NL), but also samples V801 (SR) and V269 (SR), which are incorrectly classified in this category as they are hydroponically grown tomatoes. The erroneous categorization of these two samples can be explained by the fact that these samples represent stemless tomatoes. The device also included sample V826, which represents conventionally grown tomatoes, in the triangle representing hydroponically grown tomatoes. This sample appears to contain a similar odor spectrum to hydroponically grown tomatoes. After discounting this one sample, our study indicates that e-nose has potential to be a suitable instrumental sensory analysis tool that can be used to detect hydroponically and conventionally grown crops. Similar study indicates that there is potential for the use of the e-nose to complement routine sensory analysis of tomatoes [24]. A similar view is shared by the authors [25], who state that e-nose is a fast and effective technique that does not require special sample preparation to determine the aroma of the 
product and it is therefore widely used for the detection of food adulteration. They further suggest that in the case of some foods, the aroma of the product is specific enough to distinguish the original product from its counterfeit or adulterated product. Several studies have used this fact and thus e-nose has also been used in the context of authentication and adulteration of fresh juices made from cherry tomatoes. Other authors [26] confirmed the possibility of using this device to detect adulterated food. Our results may indicate the possibility of using e-nose in the process of food authentication and in estimating food adulteration, specifically hydroponically grown tomatoes. To confirm this, we recommend working with a larger number of samples in future research.

In the past, much research has led to the development of extraction and analytical techniques useful for obtaining a detailed profile of volatiles from tomatoes. A large number of volatiles have been identified and the volatile profile of tomato has been investigated in detail in many studies. Already in 1998 [4], relationships between chemical compounds and sensory properties of tomatoes were established. (Z)-3-hexenal, hexanal, 1-octen-3one, methional, 1-penten-3-one, and 3-methylbutanal were among the most odor-active aromatic volatiles in fresh tomatoes. The aroma, taste and aftertaste of different tomato varieties were evaluated by quantitative descriptive analysis. Using PCA analysis, they found that the first three components presented $70 \%$ of the total variance. According to [5], volatiles characteristic of tomatoes include acyclic, cyclic and heterocyclic hydrocarbons, alcohols, phenols, ethers, aldehydes, ketones, carboxylic acids, esters and lactones, as well as compounds containing nitrogen, sulfur and halogens. Of these, molecules such as 1-penten-3-one, hexanal, cis-3-hexenal, trans-2-hexenal, 1-penten-3-ol, 3-methylbutanol, cis-3-hexen-1-ol, 2-isobutylthiazole, trans-2 heptanal, 6-methyl-5-hepten-2-one, 6-methyl-5hepten-2-ol, methyl salicylate, geranyl acetone, and phenyl ethanol are important.

As mentioned earlier, in this study, we focused on the five basic odors that were present in the samples in the highest amount. After the electronic nose divided the odor profile of the evaluated samples into hydroponic (inside the triangle) and conventionally grown, we were able to identify three main odor cues characteristic for hydroponically grown tomatoes (Figure 2). These are substances listed as 15.31.1-A, 26.07.1-A and, marginally, 43.88.1-A. Under the label 15.31.1-A, we are most likely to recognize the chemical organic compound 2-methylpropanol. This substance is responsible for the typical fruity flavor and the green, unripe notes of the fruit. It is highly likely that the compound dimethyl sulphide, presenting fruity and vegetal notes, also completes this set of odors. The odor substance labeled 26.07.1-A is the chemical compound 2,3-pentanedione representing a fruity flavor. This substance also promotes the freshness feeling of the fruit and participates in the formation of the sweet character. The last compound associated with the sensory character of hydroponically grown tomatoes is (Z)-3-hexen-1-ol or 1-hexanol, causing a fruity, grassy, fresh, and unripe flavor or aroma to the fruit. Correlation between (Z)-3-hexen-1-ol and flavor intensity was also proven in the study of contribution of C6 volatiles to taste and aroma [9]. Other authors report that C6 compounds are the most widespread volatiles in tomato fruit and contribute to tomato flavor in various ways. Similar to our study, the presence of (Z)-3-hexen-1-ol in hydroponically grown tomatoes was also detected by those authors [1]. In some studies, this substance is associated with consumer acceptability [27].

Therefore, our study has shown that presence of substances: 2-methylpropanol, 2,3pentanedione, (Z)-3-hexen-1-ol or 1-hexanol is potentially the difference between hydroponically and conventionally grown tomatoes. All four volatiles were also identified in a review of proven volatiles in tomatoes and tomato products in 1986 [2]. To confirm this, in future research, we recommend to upgrade research design with larger number of tested samples. In the case of machine learning analysis, we used the original electronic language dataset, whose most discriminative odorants/retention index is shown in Table 2. The dataset was then divided into $80 \%$ training data and $20 \%$ data for validation.

Using various algorithms, we found that LDA (0.879) was the most suitable, followed by Random Forrest (0.78) and SVM (0.684) using the Caret statistical package from R 4.0.2; 
KNN (0.578) were not suitable to predict hydroponic or conventional cultivation. The results including Kappa parameter are shown in Figure 3.

Table 2. Overview of retention index, sensors, and its discrimination powers (AlphaSoft 14.2 database).

\begin{tabular}{ccc}
\hline Index & Sensors & Discrimination Power \\
\hline 6 & $15.31-1-\mathrm{A}$ & 0.958 \\
16 & $26.07-1-\mathrm{A}$ & 0.951 \\
60 & $38.50-2-\mathrm{A}$ & 0.949 \\
53 & $24.41-2-\mathrm{A}$ & 0.945 \\
24 & $43.86-1-\mathrm{A}$ & 0.945 \\
52 & $23.27-2-\mathrm{A}$ & 0.942 \\
62 & $44.74-2-\mathrm{A}$ & 0.939 \\
23 & $40.49-1-\mathrm{A}$ & 0.935 \\
48 & $18.87-2-\mathrm{A}$ & 0.923 \\
51 & $22.12-2-\mathrm{A}$ & 0.915 \\
\hline
\end{tabular}

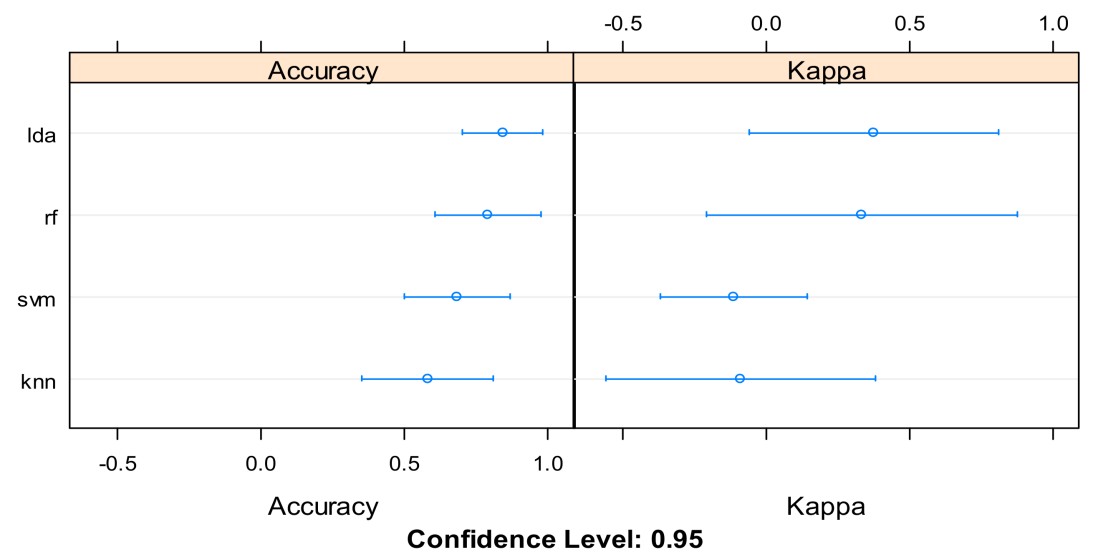

Figure 3. Machine learning applied algorithms (Caret Package, R 4.0.2).

\subsection{Sensory Analysis Carried Out by the Evaluators}

Sensory analysis can be affected by various factors. Compared to production methods, variety had greater influence on sensory attributes of tomatoes [28]. Similarly, Treftz et al. [20] reported that sensory evaluation can be influenced by several factors such as genetic composition, pre-harvest factors (light, temperature, humidity, and wind) and post-harvest factors. Other influences include price, brand, and consumer mood, which affect the sensory evaluation of the product. These authors suggest that these are all important factors to consider when evaluating the hydroponically grown product for sensory attributes. Other authors have shown that the inner tissues of tomato fruit contained higher concentrations of 3-methylbutanol, 2-methylbutanol, 3-methylbutanol, and 2-methylbutanol than pericarp [6]. The composition and types of contents in tomato were studied by some authors and they concluded that the concentration of alcohol in tomato seeds was higher than that in other tissues. This finding was focused on higher amounts of 2-methylpropanol, 3-methylbutanol, and 2-methylbutanol [29]. There is a consistent relationship between color and taste, especially in red cultivars, but according to study by Oluk et al. [30], brown cultivars were appreciated as much as their red counterparts. The brown variety had the highest sweetness, typical aroma and hardness scores, while the yellow variety had the lowest typical aroma sweetness score. In terms of sensory parameters, the red and brown varieties scored higher than the yellow and orange varieties. This means that during sensory analysis, it is important to evaluate same-color samples.

In our study, sensory panel evaluated homogenic-looking samples and the evaluation was divided into two parts. In the first part of the sensory evaluation, a 5-level scoring 
test was used, which focused on the more complex agricultural character of tomatoes. The results obtained were analyzed in SensoMiner software, based on which we obtained a graphical representation of the different attributes and the results obtained are shown in the form of Figure 4. From this graphical representation, we can see that in the odor attribute, samples V102 (NL) and V153 (CZ) were judged as the least intense and they achieved worse evaluation. Medium values were assigned by the evaluators to samples representing conventionally grown tomatoes: V188 (NL), V297 (BE), and V826 (IT). Of all the samples evaluated, no sample proved to be dominantly more intense in the odor attribute compared to the other samples. Samples representing hydroponically grown tomatoes: V269 (SR), V512 (CZ), V740 (SR), and V931 (SR) were ranked with the highest values obtained, which means that they showed a more intense odor. These results correspond with those obtained using e-nose.

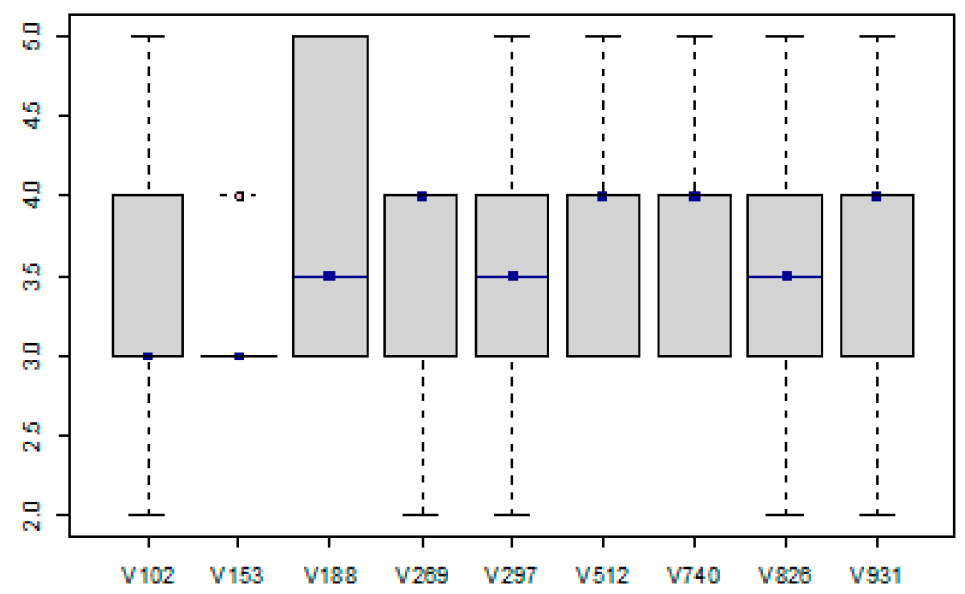

Figure 4. Sensory analysis of tomato fruit odor.

Another attribute evaluated by the sensory panel was the smell of the tomato fruit on the cut. From the data shown in the form of Figure 5, we can see that there were no clear worse or better results between the samples. Samples V188 (NL), V269 (SR), V512 (Strabena, CZ), V740 (SR), and V931 (SR) were evaluated as more satisfactory by the sensory panel in terms of odor on the cut. In contrast, samples V102 (NL), V153 (CZ), and V826 (IT) were characterized by a less intense aroma profile. In terms of the attribute evaluated, sample V297, which represents conventionally grown Belgian tomatoes, was rated as the most pleasant. The graphical distribution of samples V512 (CZ) and V826 (IT) tells us that these samples had a wide odor spectrum.

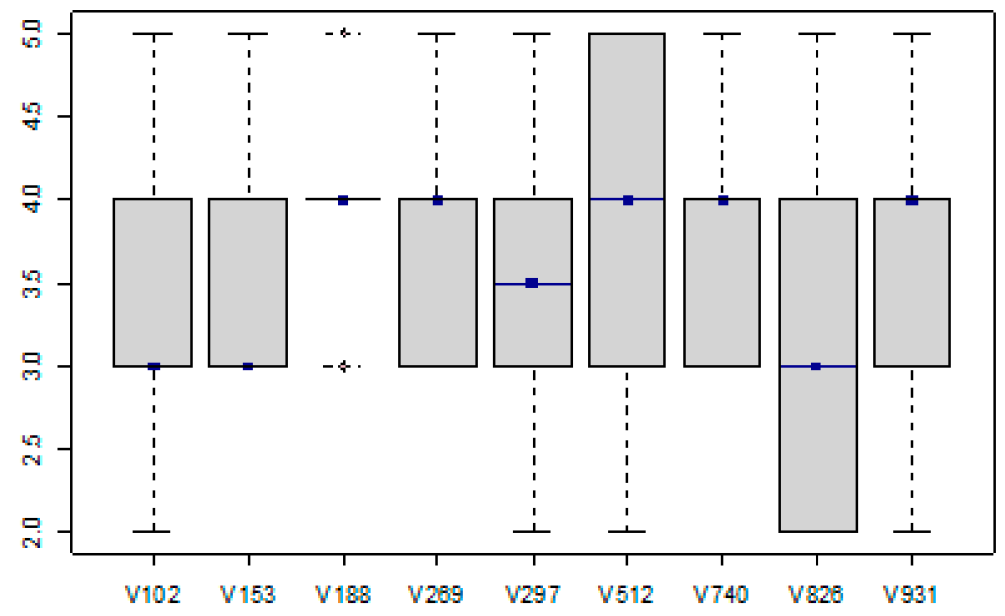

Figure 5. Sensory analysis of the smell of fruit on the cut. 
The human perception of tomato flavor lies in the integration of taste and aroma. Tomato aroma is a balance of acid and sugar recognized by the tongue and the effect of volatile compounds in the fruit that cause the aroma is recognized by the nose, therefore, we considered important the results obtained by sensory evaluation of tomato flavor, which are graphically represented in the form of Figure 6. For the flavor attribute, the sample V153 (CZ) stood out as more intense. This sample received, on average, the highest possible score and it is the sample that represents hydroponically grown tomatoes. Compared to this sample, samples V188 (NL), V512 (CZ), and V931 (SR) were rated as less intensive. These samples represent the tomatoes of both groups. The sample that was rated lowest by the sensory panel and rated as less suitable for this attribute was sample V269 (SR). The other samples were at the level of average taste acceptable by consumers.

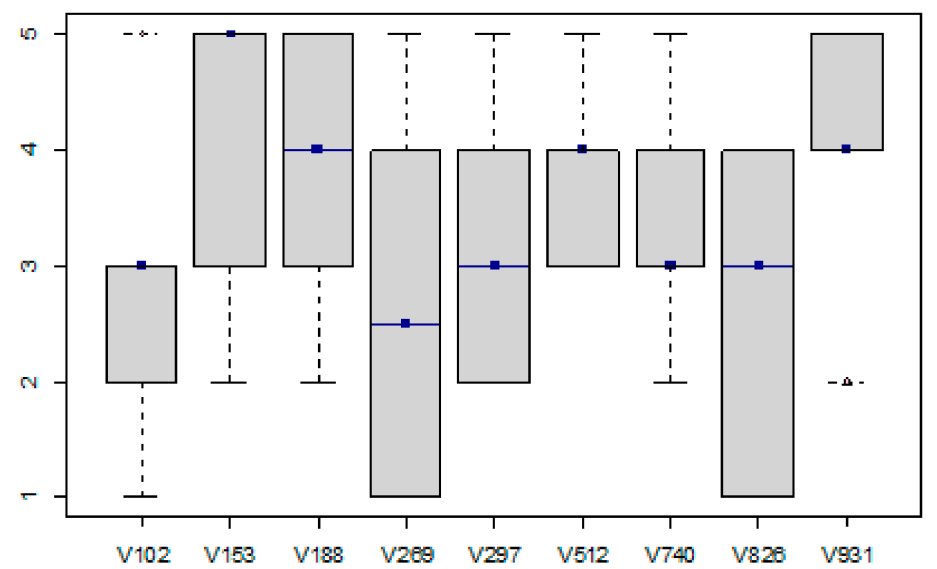

Figure 6. Sensory analysis of the flavor of the fruit.

The results obtained by the scoring test were subjected to PCA analysis and Figure 7 was constructed. The first part of Figure 7a shows the distribution of the samples within the summary scores of all the attributes evaluated, namely: appearance, smell, smell of the fruit on the cut, texture, taste, and overall impression. Based on the data obtained from the sensory analysis, we can rank samples V269 (SR) and V297 (BE), as well as samples V102 (NL) and V826 (IT) as statistically significantly similar. The remaining evaluated samples scored similarly, meaning that these samples show similar sensory characteristics. Based on the samples' position within the graph, we can conclude that the sensory evaluators were also able to detect differences between hydroponic and conventional tomatoes. This means that also through the panelists' evaluation with the simple scoring test and subsequent PCA analysis, it is possible to show differences between tomatoes grown in two different ways. Other authors have compared hydroponically and conventionally grown crops from sensory point of view. For example, in one study, the authors compared hydroponically and conventionally grown strawberries. The study found that consumers did not have strong preference between hydroponic and soil-grown strawberries, but indicated that of the 13 attributes examined, overall aroma and aroma intensity were the only attributes that reached statistical significance $(p<0.05)$. Hydroponically grown strawberries showed higher mean ratings for these two categories. Consequently, they reported that due to the environmental benefits of hydroponic production combined with the favorable ratings of descriptive sensory analysis, it may be desirable for the consumer and beneficial for the environment to grow strawberry varieties in infertile areas to provide fresh fruit [20]. In 2011, a study was conducted to compare hydroponically, conventionally, and organically grown lettuces. A significant difference was found between either hydroponically and conventionally grown lettuces $(p=0.03)$ or between organically and conventionally grown lettuces $(p=0.009)$, but not between hydroponically and organically grown lettuces $(p=0.6956)$. In the opinion of the sensory panel, hydroponically and organically grown lettuces had more intense smell (odor) [31]. Similarly, in our study, consumers rated hydroponically grown 
tomatoes with the highest scores, meaning that they demonstrated a more intense odor. These results were confirmed by e-nose.

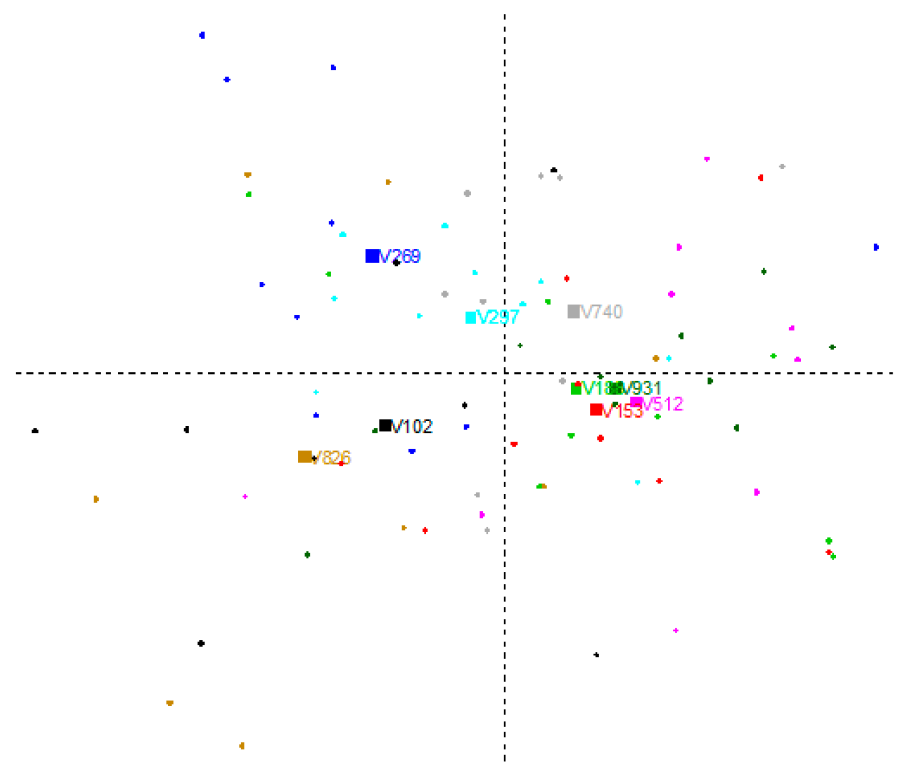

(a)

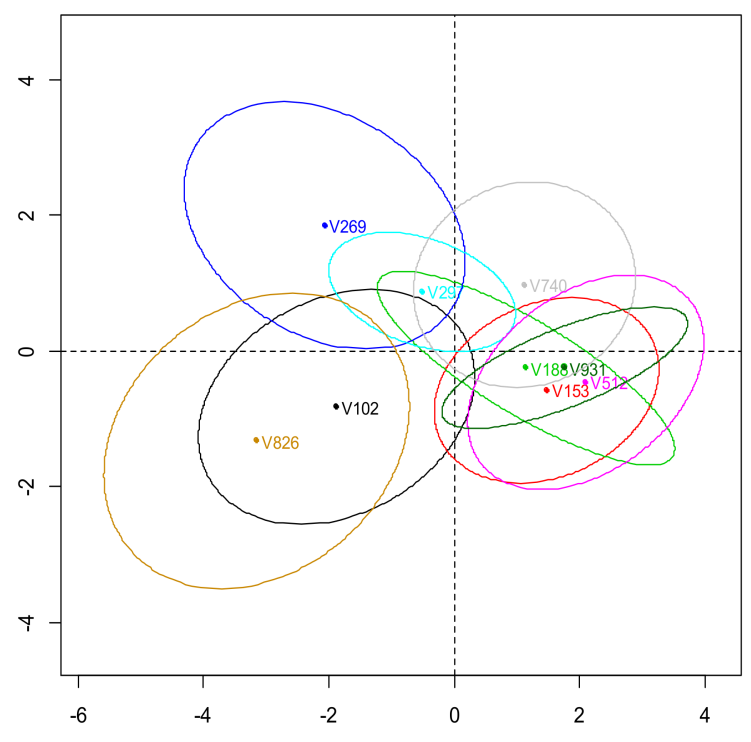

(b)

Figure 7. (a) PCA analysis of the overall character of the samples (point test); (b) PCA analysis of the complex profile of the samples (point test)—output by Sensominer software.

Within the second part of Figure $7 \mathrm{~b}$, the ellipses shown represent the variance of the values given in the sensory evaluation of each attribute. We can say that the samples V297 (BE) and V931 (SR) have the narrowest variance of the values obtained. A very wide variance is visible for samples V826 (IT), V102 (NL), and V269 (SR).

Table 3 was subsequently generated from the results of the scoring test, showing the demonstrability of differences between the tested samples. The resulting values, which are highlighted in yellow in the table, indicate that there is a difference between them at the alpha level of significance $=0.05$. For us, the most important results are indicated in bold. Sample V826 (IT), which belongs to conventionally grown tomatoes, was marked as statistically significantly different. Its dissimilarity was not proven for samples V102 (NL) and V269 (SR). This means that these samples had similar sensory characteristics to the tomatoes that were stemless. The Slovak hydroponically grown tomatoes V931 were statistically significantly different from other samples, namely samples V102 (NL), V297 (BE), and V826 (IT). Similarly, to the Slovak sample, the Czech tomato (V512) differed from the samples that were grown conventionally (V102, V297, and V826).

Table 3. Demonstrability of differences between samples (sensory evaluation) (yellow label statistical difference $p<0.05)$.

\begin{tabular}{llllllllll}
\hline & V102 & V153 & V188 & V269 & V297 & V512 & V740 & V826 & V931 \\
\hline V102 & 1 & 0.0549 & 0.0985 & 0.0804 & 0.1240 & 0.0279 & 0.0550 & 0.6970 & 0.0481 \\
V153 & & 1 & 0.9235 & 0.00833 & 0.0350 & 0.8637 & 0.2268 & 0.0115 & 0.8957 \\
V188 & & & 1 & 0.0718 & 0.3100 & 0.7846 & 0.3827 & 0.0207 & 0.8946 \\
V269 & & & & 1 & 0.3465 & 0.0050 & 0.0666 & 0.0550 & 0.0057 \\
V297 & & & & & 1 & 0.0206 & 0.3057 & 0.0406 & 0.0144 \\
V512 & & & & & & 1 & 0.2068 & 0.0063 & 0.8747 \\
V740 & & & & & & & 1 & 0.0127 & 0.2043 \\
V826 & & & & & & & & 1 & 0.0106 \\
V931 & & & & & & & & & \\
\hline
\end{tabular}


The second part of the sensory analysis performed by the sensory panel consisted of assessing the sensory profiles through the hedonic scale. Our evaluators assessed the odor-flavor profile of the tomato, i.e., the flavor. Flavor refers to the sensory impression of the food or other substance, which is determined mainly by chemical combinations of taste and smell. To obtain the results, we used a similar program to the scoring test, namely SensoMiner, and we provide the results by visualizing them in the table evaluating the treated averages of the measured values. From a statistical point of view, we used Friedman's non-parametric test. The provable differences between the samples are demonstrated to us by the values shown in Table 4 . Statistically demonstrably stronger, more pronounced attributes are shown in blue. Attributes in which specific samples were rated as statistically demonstrably weaker are shown in yellow. From the values shown, we can see that in terms of sweet flavor, tomato sample with stem V931, hydroponically grown in Slovakia, dominates. The evaluators identified the foreign, conventionally grown samples V297 (BE) and V826 (IT) as statistically significantly less sweet. In this context, lower fruit ripeness was also associated with sample V826 and a demonstrably more acidic flavor with sample V297. Sample V740 (SR) was also found to be sour. Sample V269 (SR) was less acidic than the other samples. In terms of the other evaluated flavors, there was no clear difference between the samples. This means that there were no spicy, peppery, hot, metallic, or other foreign odors or flavors present in the samples that would significantly affect the character of the tomatoes. As a result, it can be said that samples V297 (BE) and V826 (IT), which represented foreign conventionally grown tomato varieties, were marked as less ripe or underripe by this method of evaluation and were therefore less sweet but more acidic to the evaluators compared to other samples. These results can be explained with the findings from previous studies that have shown that tomato volatiles which affect odor and taste of the tomato are formed during the ripening process in the intact tomato and during maceration of the tomato, for example, when slicing, chewing or blending. A reasonable amount of cis-3-hexenal also exists in the ripe green tomato, but its concentration increased more than 20 times in the ripe form [11]. These samples were from abroad and had to be shipped to Slovakia; they may be chilled during the transportation and as a result of that, their volatile content may have been reduced as shown in the study by Tieman et al. [9] where refrigeration of tomatoes have previously been found to reduce the volatile content of fresh tomato fruit. It has been reported that chilling injury results in membrane damage, which can disrupt the lipoxygenase enzymatic pathway that results in C-6 aldehydes (hexanal, hexanol, cis-3-hexenal, cis-3-hexenol, trans-2-hexenal, etc.) from membrane lipids [8]. On the other hand, Yilmaz [32] gave a list of volatiles whose amount increases after disruption of tissues (cis-2-hexenal, trans-2-hexenal, hexanal, trans-2-heptenal, 1-penten-3-one, 1-penten-3-ol, and geranyl acetone).

Table 4. PCA analysis of sensory profiles (blue label statistically better, yellow label vice versa).

\begin{tabular}{lllllll}
\hline & Sweet Flavor & Ripe/Overripe & Sour Flavor & $\begin{array}{l}\text { Peppery/Hot/Spicy } \\
\text { Flavor }\end{array}$ & Metallic Flavor & $\begin{array}{l}\text { Other Foreign } \\
\text { Flavor }\end{array}$ \\
\hline V931 & 7.52 & 6.17 & 4.46 & 1.58 & 1.15 & 0.59 \\
V102 & 5.56 & 6.14 & 3.33 & 1.39 & 1.74 & 1.43 \\
V512 & 5.48 & 5.20 & 4.35 & 2.30 & 0.91 & 0.89 \\
V153 & 6.02 & 5.21 & 3.99 & 1.62 & 2.14 & 0.92 \\
V188 & 6.17 & 5.80 & 3.68 & 2.21 & 1.73 & 1.41 \\
V740 & 4.46 & 5.42 & 6.22 & 2.15 & 1.02 & 0.85 \\
V269 & 4.48 & 6.67 & 2.63 & 1.55 & 2.28 & 2.00 \\
V297 & 2.36 & 4.85 & 6.55 & 2.16 & 2.32 & 1.64 \\
V826 & 3.10 & 2.46 & 4.11 & 1.98 & 2.20 & 2.00 \\
\hline
\end{tabular}

The demonstrability of the differences from the results of the sensory profile evaluations at alpha level $=0.05 \%$ is shown in Table 5 . The sample V826 (IT) was also the most different according to this analysis. This sample is statistically significantly different from 
all samples except the Dutch stemless tomatoes. Tomato sample V740 (SR) was significantly different from sample V153 (CZ) and from V269 (SR).

Table 5. Demonstrability of differences between samples (sensory profiles) (yellow label statistical difference $p<0.05)$.

\begin{tabular}{llllllllll}
\hline & V102 & V153 & V188 & V269 & V297 & V512 & V740 & V826 & V931 \\
\hline V102 & 1 & 0.9121 & 0.9857 & 0.9211 & 0.0379 & 0.6828 & 0.1126 & 0.0504 & 0.6559 \\
V153 & & 1 & 0.9365 & 0.6461 & 0.0658 & 0.8984 & 0.0470 & 0.0078 & 0.3164 \\
V188 & & & 1 & 0.7963 & 0.0737 & 0.8130 & 0.0917 & 0.0176 & 0.5927 \\
V269 & & & & 1 & 0.0049 & 0.2519 & 0.0181 & 0.0234 & 0.4146 \\
V297 & & & & & 1 & 0.0749 & 0.3593 & 0.0106 & 0.0042 \\
V512 & & & & & & 1 & 0.1121 & 0.0114 & 0.1929 \\
V740 & & & & & & & 1 & 0.0000 & 0.0374 \\
V826 & & & & & & & & 1 & 0.0008 \\
V931 & & & & & & & & & 1 \\
\hline
\end{tabular}

The similarity between the samples was then also analyzed using PCA method and a similarity map of the samples was constructed (Figure 8). An extract from Figure 8a shows the individual description and distribution of the samples through a complex odor profile by combining the individual flavors assessed. The resulting position of the samples in the graph indicates that samples V740 (SR) and V297 (BE) are rated as statistically demonstrably similar, meaning that these samples are similar in their odor profile composition. Since sample V826 (IT) is located in a separate quarter, we can conclude that this sample is statistically significantly different from the other samples. In the graph, the other samples are placed close to each other, indicating that these samples are very similar.

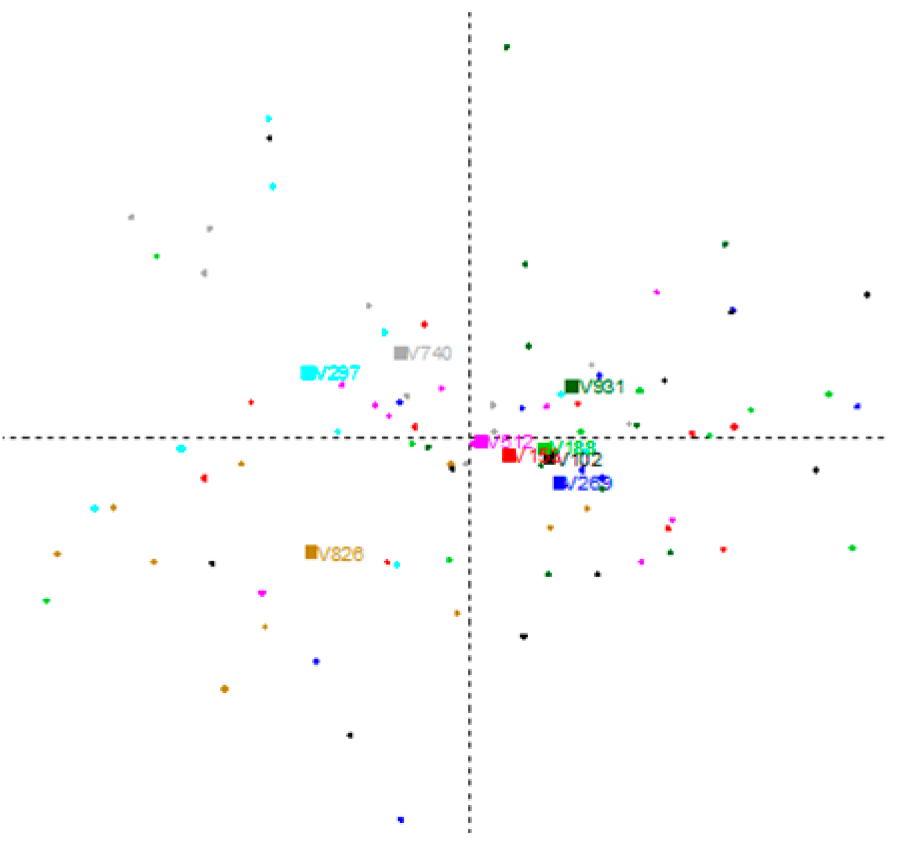

(a)

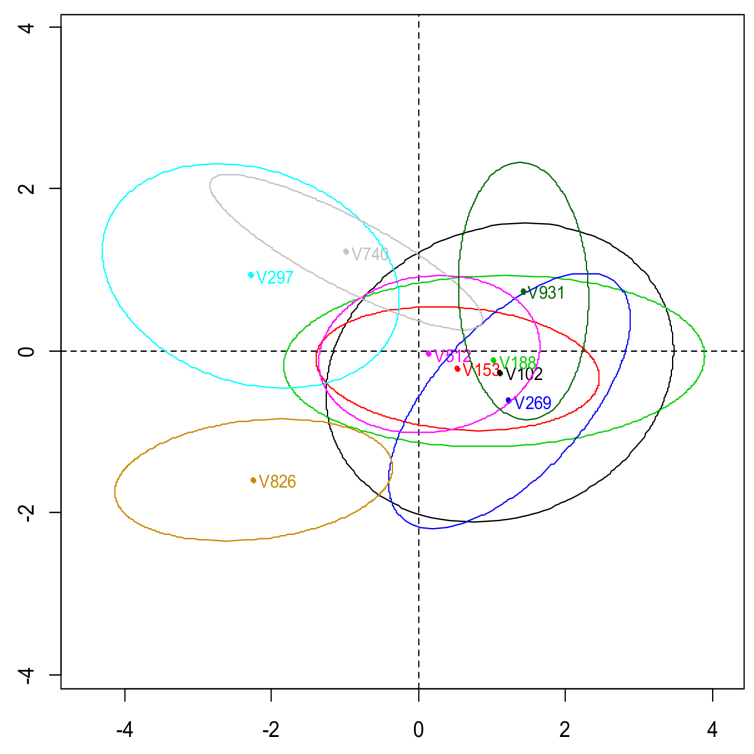

(b)

Figure 8. (a) PCA analysis of the flavor assessment of samples (sensory profiles); (b) PCA analysis of the complex profile of samples (sensory profiles).

A PCA similarity map was also constructed (Figure 8b), which shows the variances obtained from the results of the sensory profile evaluations. In this case, each ellipse represents values from minimum to maximum. Sample V826 (IT) also emerged as statistically demonstrably different from the other samples in this case, which does not have 
such a large variance, but apparently quite different sensory properties, as we have confirmed in the previous results. We observe a similar flavor in samples V740 (SR) and V297 (BE). Among the group of samples with related characteristics, the conventionally grown samples from the Netherlands (V102 and V188) have a broad profile.

In the analysis of the relationship between the two types of measurements, we can conclude that there is no relationship between the data from the electronic nose to the data from the sensory evaluation. The odor profile is complex in both types of tomato grown and the two methods do not correlate with each other at all $(R=0.07)$. The results are demonstrated in Figure 9.
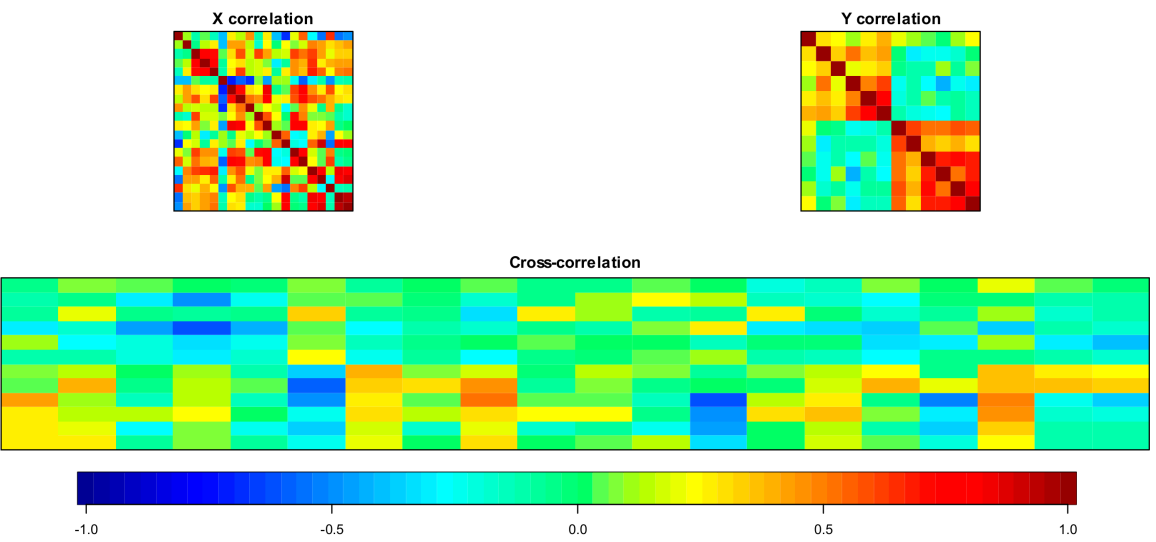

Figure 9. Canonical correlation analysis $\mathrm{X}$ matrix (electronic nose data, $\mathrm{R}=0.34$ ), $\mathrm{Y}$ matrix (sensory analysis data, $R=0.22$ ), bottom $X Y$ (cross-correlation matrix of $X Y$ data, $R=0.07$ ).

\section{Conclusions}

Sensory analysis of hydroponically and conventionally grown tomatoes was carried out using e-nose and sensory panel. In both cases, there were demonstrable differences between the two groups and the study proved that there is the difference between tomatoes grown with nutrient solution and those grown conventionally in soil. Based on the obtained results, we can conclude that we can potentially use the electronic nose to detect the difference between the samples. E-nose device was able to divide the analyzed samples into two groups, namely hydroponically and conventionally grown tomatoes. This fact shows us that we could potentially use e-nose for detecting food adulteration, namely, conventionally grown tomatoes declared as hydroponically grown tomatoes and vice versa. For future research, to obtain more accurate results, we recommend testing a larger number of samples and purchasing samples from one location or country.

In our research, we were also able to identify three main odor traces characteristic for hydroponically grown tomatoes: 2-methylpropanol, 2,3-pentanedione, (Z)-3-hexen-1-ol or 1-hexanol (substances registered as 15.31.1-A, 26.07.1-A and, marginally, 43.88.1-A). It is very likely that these substances characterize the difference between tested samples. Sensory panel was also able to detect the difference between hydroponically and conventionally grown tomatoes, which tells us that even consumers themselves are able to recognize the differences between the two groups. For the prediction of hydroponically and convectively grown tomatoes, we used a machine learning technique (ML), with the linear LDA algorithm performing best. There was no relationship between the sensory evaluation data and the artificial perception evaluation.

Author Contributions: Conceptualization, N.V., V.V. and J.Š.; methodology, N.V., V.V., P.M. and J.Š.; software, V.V. and A.M.; validation, V.V. and M.K.; formal analysis, N.V., J.Š. and P.M.; investigation, M.K., V.V. and N.V.; resources, M.K., V.V., N.V. and A.M.; data curation, J.Š., P.M. and V.V.; writingoriginal draft preparation, M.K., V.V. and A.M.; writing-review and editing, M.K., A.M. and V.V.; visualization, V.V., A.M., J.Š. and N.V.; supervision, J.Š. and V.V. All authors have read and agreed to the published version of the manuscript. 
Funding: The research was also funded by the research grant APVV-17-0564 "The Use of Consumer Neuroscience and Innovative Research Solutions in Aromachology and its Application in Production, Business and Services".

Institutional Review Board Statement: Not applicable.

Informed Consent Statement: Not applicable.

Data Availability Statement: Not applicable.

Conflicts of Interest: The authors declare no conflict of interest.

\section{References}

1. Jukić Špika, M.; Dumičić, G.; Brkić Bubola, K.; Soldo, B.; Goreta Ban, S.; Vuletin Selak, G.; Ljubenkov, I.; Mandušić, M.; Žanić, K. Modification of the Sensory Profile and Volatile Aroma Compounds of Tomato Fruits by the Scion $\times$ Rootstock Interactive Effect. Front. Plant Sci. 2021, 11, 616431. [CrossRef] [PubMed]

2. Petro-Turza, M. Flavor of tomato and tomato products. Food Rev. Int. 1986, 2, 309-351. [CrossRef]

3. Buttery, R.G.; Teranishi, R.; Ling, L.C. Fresh tomato aroma volatiles: A quantitative study. J. Agric. Food Chem. 1987, 35, 540-544. [CrossRef]

4. Krumbein, A.; Auerswald, H. Characterization of aroma volatiles in tomatoes by sensory analyses. Food Nahr. 1998, 42, 395-399. [CrossRef]

5. Paolo, D.; Bianchi, G.; Lo Scalzo, R.; Morelli, C.F.; Rabuffetti, M.; Speranza, G. The Chemistry behind Tomato Quality. Nat. Prod. Commun. 2018, 13, 1225-1232. [CrossRef]

6. Wang, L.; Qian, C.; Bai, J.; Luo, W.; Jin, C.; Yu, Z. Difference in volatile composition between the pericarp tissue and inner tissue of tomato (Solanum lycopersicum) fruit. J. Food Process Preserv. 2017, 42, e13387. [CrossRef]

7. Wu, Q.; Tao, X.Y.; Ai, X.Z.; Luo, Z.S.; Mao, L.C.; Ying, T.J.; Li, L. Effect of exogenous auxin on aroma volatiles of cherry tomato (Solanum lycopersicum L.) fruit during postharvest ripening. Postharvest Biol. Technol. 2018, 146, 108-116. [CrossRef]

8. Baldwin, E.A.; Goodner, K.; Plotto, A. Interaction of Volatiles, Sugars, and Acids on Perception of Tomato Aroma and Flavor Descriptors. J. Food Sci. 2008, 73, 294-307. [CrossRef]

9. Tieman, D.; Bliss, P.; McIntyre, L.M.; Blandon-Ubeda, A.; Bies, D.; Odabasi, A.Z.; Rodríguez, G.R.; der Knaap, E.; Taylor, M.G.; Goulet, C.H.; et al. The Chemical Interactions Underlying Tomato Flavor Preferences. Curr. Biol. 2012, 22, 1035-1039. [CrossRef]

10. Davidovich-Rikanati, R.; Sitrit, Y.; Tadmor, Y.; Pichersky, E.; Dudareva, N.; Lewinsohn, E. Tomato Aroma: Biochemistry and Biotechnology. In Biotechnology in Flavor Production, 2nd ed.; Havkin-Frenkel, D., Dudai, N., Eds.; Wiley-Blackwell: Hoboken, NJ, USA, 2016; pp. 243-263.

11. Carbonell-Barrachina, A.A.; Agustí, A.; Ruiz, J.J. Analysis of flavor volatile compounds by dynamic headspace in traditional and hybrid cultivars of Spanish tomatoes. Eur. Food Res. Technol. 2006, 222, 536-542. [CrossRef]

12. Causse, M.; Saliba-Colombani, V.; Lesschaeve, I.; Buret, M. Genetic analysis of organoleptic quality in fresh market tomato. 2. Mapping QTLs for sensory attributes. Theor. Appl. Genet. 2001, 102, 273-283. [CrossRef]

13. Baldwin, E.; Plotto, A.; Narciso, J.; Bai, J. Effect of 1-methylcyclopropene on tomato flavour components, shelf life and decay as influenced by harvest maturity and storage temperature. J. Sci. Food Agric. 2011, 91, 969-980. [CrossRef]

14. Sato, S.; Sakaguchi, S.; Furukawa, H.; Ikeda, H. Effects of $\mathrm{NaCl}$ application to hydroponic nutrient solution on fruit characteristics of tomato (Lycopersicon esculentum Mill.). Sci. Hortic. 2006, 109, 248-253. [CrossRef]

15. Treftz, C.; Omaye, S.T. Hydroponics: Potential for augmenting sustainable food production in non-arable regions. Nutr. Food Sci. 2016, 46, 672-684. [CrossRef]

16. Sanjuan-Delmás, D.; Josa, A.; Muñoz, P.; Gassó, S.; Rieradevall, J.; Gabarrell, X. Applying nutrient dynamics to adjust the nutrient-water balance in hydroponic crops. A case study with open hydroponic tomato crops from Barcelona. Sci. Hortic. 2020, 261, 108908. [CrossRef]

17. Verdoliva, S.G.; Gwyn-Jones, D.; Detheridge, A.; Robson, P. Controlled comparisons between soil and hydroponic systems reveal increased water use efficiency and higher lycopene and $\beta$-carotene contents in hydroponically grown tomatoes. Sci. Hortic. 2021, 279, 109896. [CrossRef]

18. Schmautz, Z.; Loeu, F.; Liebisch, F.; Graber, A.; Mathis, A.; Griessler Bulc, T.; Junge, R. Tomato Productivity and Quality in Aquaponics: Comparison of Three Hydroponic Methods. Water 2016, 8, 533. [CrossRef]

19. Morgan, L. Hydroponic Tomatoes. GPN 2003, 78-85. Available online: http://gpnmag.com/wp-content/uploads/p78\%20 Morgan.pdf (accessed on 6 June 2021).

20. Treftz, C.H.; Zhang, F.; Omaye, S.T. Comparison between Hydroponic and Soil-Grown Strawberries: Sensory Attributes and Correlations with Nutrient Content. Food Nutr. Sci. 2015, 6, 1371-1380. [CrossRef]

21. Karakaya, D.; Ulucan, O.; Turkan, M. Electronic Nose and Its Applications: A Survey. Int. J. Autom. Comput. 2020, 17, 179-209. [CrossRef]

22. Park, S.Y.; Kim, Y.; Kim, T.; Eom, T.H.; Kim, S.Y.; Jang, H.W. Chemoresistive materials for electronic nose: Progress, perspectives, and challenges. InfoMat 2019, 1, 289-316. [CrossRef] 
23. Marsili, R. Combining mass spectrometry and multivariate analysis to make a reliable and versatile electronic nose. In Flavor, Fragance and Odor Analysis; Marsili, R., Ed.; CRC Press: Boca Raton, FL, USA, 2002; pp. 349-374.

24. Berna, A.Z.; Buysens, S.; Natale, C.D.; Grün, I.U.; Lammertyn, J.; Nicolaï, B.M. Relating sensory analysis with electronic nose and headspace fingerprint MS for tomato aroma profiling. Postharvest Biol. Technol. 2005, 36, 143-155. [CrossRef]

25. Gliszczyńska-Świgło, A.; Chmielewski, J. Electronic Nose as a Tool for Monitoring the Authenticity of Food. A Review. Food Anal. Methods 2016, 10, 1800-1816. [CrossRef]

26. Hong, X.; Wang, J. Detection of adulteration in cherry tomato juices based on electronic nose and tongue: Comparison of different data fusion approaches. J. Food Eng. 2014, 126, 89-97. [CrossRef]

27. Pichersky, E.; Noel, J.P.; Dudareva, N. Biosynthesis of Plant Volatiles: Nature's Diversity and Ingenuity. Science 2006, 311, 808-811. [CrossRef]

28. Johanson, L.; Haglund, Å.; Berglund, L.; Lea, P.; Risvik, E. Preference for tomatoes, affected by sensory attributes and information about growth conditions. Food Qual. Prefer. 1999, 10, 289-298. [CrossRef]

29. Li, J.; Di, T.; Bai, J. Distribution of Volatile Compounds in Different Fruit Structures in Four Tomato Cultivars. Molecules 2019, 24, 2594. [CrossRef]

30. Oluk, A.C.; Ata, A.; Ünlü, M.; Yazici, E.; Karaşahin, Z.; Erogulu, E.Ç.; Canan, İ. Biochemical Characterisation and Sensory Evaluation of Differently Coloured and Shaped Tomato Cultivars. Not. Bot. Horti Agrobot. Cluj-Napoca 2019, 47, 599-607. [CrossRef]

31. Murphy, M.T.; Zhang, F.; Nakamura, Y.K.; Omaye, S.T. Comparison between Hydroponically and Conventionally and Organically Grown Lettuces for Taste, Odor, Visual Quality and Texture: A Pilot Study. Food Nutr. Sci. 2011, 2, 124-127. [CrossRef]

32. Yilmaz, E. The chemistry of fresh tomato flavor. Turk. J. Agric. For. 2001, 25, 149-155. 